\title{
Communication: Hydration structure and polarization of heavy alkali ions: A first principles molecular dynamics study of $\mathrm{Rb}^{+}$and $\mathrm{Cs}^{+}$
}

Takashi Ikeda, and Mauro Boero

Citation: The Journal of Chemical Physics 137, 041101 (2012); doi: 10.1063/1.4742151

View online: https://doi.org/10.1063/1.4742151

View Table of Contents: http://aip.scitation.org/toc/jcp/137/4

Published by the American Institute of Physics

\section{Articles you may be interested in}

Hydration of alkali ions from first principles molecular dynamics revisited

The Journal of Chemical Physics 126, 034501 (2007); 10.1063/1.2424710

Hydration properties of magnesium and calcium ions from constrained first principles molecular dynamics The Journal of Chemical Physics 127, 074503 (2007); 10.1063/1.2768063

Role of van der Waals corrections in first principles simulations of alkali metal ions in aqueous solutions The Journal of Chemical Physics 143, 194510 (2015); 10.1063/1.4935932

Revisiting the hydration structure of aqueous $\mathrm{Na}^{+}$

The Journal of Chemical Physics 146, 084504 (2017); 10.1063/1.4975608

First-principles-based simulation of interlayer water and alkali metal ions in weathered biotite

The Journal of Chemical Physics 145, 124703 (2016); 10.1063/1.4963017

Perspective: How good is DFT for water?

The Journal of Chemical Physics 144, 130901 (2016); 10.1063/1.4944633

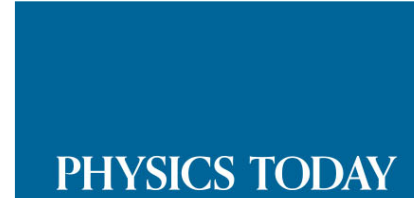

WHITEPAPERS
ADVANCED LIGHT CURE ADHESIVES

READ NOW

Take a closer look at what these environmentally friendly adhesive systems can do
PRESENTED BY

8) MASTERBOND 


\title{
Communication: Hydration structure and polarization of heavy alkali ions: A first principles molecular dynamics study of $\mathbf{R b}^{+}$and $\mathbf{C s}^{+}$
}

\author{
Takashi Ikeda ${ }^{1, a)}$ and Mauro Boero ${ }^{2}$ \\ ${ }^{1}$ Condensed Matter Science Division, Quantum Beam Science Directorate, Japan Atomic Energy Agency \\ (JAEA), 1-1-1 Kouto, Sayo, Hyogo 679-5148, Japan \\ ${ }^{2}$ Institut de Physique et Chimie des Matériaux de Strasbourg, UMR 7504 CNRS-University of Strasbourg, \\ 23 rue du Loess, 67034 Strasbourg, France
}

(Received 20 June 2012; accepted 19 July 2012; published online 31 July 2012)

\begin{abstract}
Hydration structure and polarization of $\mathrm{Rb}^{+}$and $\mathrm{Cs}^{+}$in liquid water at ambient conditions were studied by first principles molecular dynamics. Our systematic analysis of the relevant electronic structures, based on maximally localized Wannier functions, revealed that the dipole moment of $\mathrm{H}_{2} \mathrm{O}$ molecules in the first solvation shell of the ions slightly increases with increasing the atomic number. We also found that the polarization of heavy alkali ions, particularly $\mathrm{Cs}^{+}$, tends to stabilize a peculiar asymmetric hydration structure with relevant consequences in the extraction of the harmful ${ }^{137} \mathrm{Cs}$ resulting from nuclear wastes. () 2012 American Institute of Physics. [http://dx.doi.org/10.1063/1.4742151]
\end{abstract}

Understanding the Hofmeister series, which is a classification of ions according to their ability to structurize water (or their affinity to bind to proteins and nucleic acids), is a long standing research target in many areas at the crossroad among physics, chemistry, and biology. Nonetheless, the mechanism leading to enhancement or weakening of the hydrogen bond (H-bond) network has not been entirely elucidated. Experimentally, the hydration of metal ions has been investigated using elaborate techniques which have provided fundamental pieces of information, such as ion-water distance, hydration structure, and dynamics of hydrated ions, as shown in renowned monographs ${ }^{1-4}$ and review articles. ${ }^{5,6}$

Besides the evident physico-chemical importance, selectivity of alkali ions is a crucial issue also in biology and environmental sciences. Specifically, understanding adsorption and desorption processes of highly soluble radionuclides, such as ${ }^{137} \mathrm{Cs}$, in clays has become one of the urgent requests in order to develop efficient extractors of radionuclides from contaminated clay minerals, as the recent accident occurred to the Fukushima dai-ichi nuclear plant in Japan has proven. ${ }^{7,8}$ For most clays, the observed Hofmeister series is $\mathrm{Cs}^{+}>\mathrm{K}^{+}$ $>\mathrm{Na}^{+}>\mathrm{Li}^{+}$, indicating that the affinity of the ions increases with the (non-hydrated) ionic radius. ${ }^{9}$ However, this observation cannot be fully rationalized by simply considering the crude electrostatic interaction, which would actually favor smaller unhydrated ions. Therefore it is clear that a yet unidentified quantum mechanical interaction comes into play, along with electrostatics, enhancing the affinity of heavy alkali ions to clay minerals.

In our previous works, ${ }^{10-12}$ hydration of $\mathrm{Li}^{+}, \mathrm{Na}^{+}$, and $\mathrm{K}^{+}$was investigated by first principles molecular dynamics (FPMD), providing detailed insight not only into the interplay between solutes and solvents, but also into the strict relationships among molecular orientation, $\mathrm{H}$-bond formation polar-

\footnotetext{
${ }^{a)}$ Electronic mail: ikeda.takashi@jaea.go.jp.
}

ization of hydration waters, and infrared spectra. The scope of this work is, on one hand, to examine whether the observed trends still hold for heavier alkali ions, and, on the other hand, to inspect the hydration behavior specific to heavy alkali ions, along with its related electronic properties, which are likely to be responsible for the mentioned selectivity of heavy alkali ions.

We performed Car-Parrinello ${ }^{13}$ FPMD simulations ${ }^{14}$ of alkali ions in liquid water at ordinary density $\left(1.0 \mathrm{~g} / \mathrm{cm}^{3}\right)$ and temperature $(T=300 \mathrm{~K})$, within the density functional theory ${ }^{15}$ (DFT) approach, including the generalized gradient approximation (GGA) to exchange and correlation after Hamprecht-Cohen-Tozer-Handy ${ }^{16}$ (HCTH), previously benchmarked for our target. ${ }^{10,17-19}$ The valence-core interaction was described by a von Barth-Car pseudopotential ${ }^{20}$ for $\mathrm{H}$, a norm-conserving Troullier-Martins pseudopotential ${ }^{21}$ for $\mathrm{O}$, and a Goedecker-Teter-Hutter type pseudopotential ${ }^{22,23}$ for $\mathrm{Rb}$ and $\mathrm{Cs}$, in which semicore states were included as valence states. Valence orbitals were expanded in a plane wave (PW) basis set with an energy cutoff of $80 \mathrm{Ry}$. The Brillouin zone was sampled at the $\Gamma$ point only. The equations of motion were integrated with a time step of 4 a.u. (0.0968 fs) and a fictitious electron mass of 400 a.u. ensuring reliable static and dynamical properties ${ }^{24,25}$ and a good adiabaticity of the Car-Parrinello trajectories. ${ }^{26}$ Our approach was validated by examining the ion-oxygen distances $d$ and related hydration energies $\Delta E$ for $\mathrm{M}^{+} \mathrm{H}_{2} \mathrm{O}$ complexes $(\mathrm{M}=\mathrm{Rb}$ and Cs). Our estimated $d$ and $\Delta E$ of 2.830 (3.013) $\AA$ and 14.7 (13.2) $\mathrm{kcal} / \mathrm{mol}$ for $\mathrm{Rb}^{+} \mathrm{H}_{2} \mathrm{O}\left(\mathrm{Cs}^{+} \mathrm{H}_{2} \mathrm{O}\right)$, respectively, agree well with the corresponding estimate of 2.763 (2.962) $\AA$ and $16.3(13.5) \mathrm{kcal} / \mathrm{mol}$ made with a standard quantum chemistry method at the MP2 level of theory. ${ }^{27,28}$

Our $\mathrm{Rb}^{+}$and $\mathrm{Cs}^{+}$aqueous solution systems are similar to the ones used in our former studies. ${ }^{10,17}$ Specifically, we used a cubic simulation cell of side $L=12.417 \AA$ in which the metal cation was solvated by $63 \mathrm{H}_{2} \mathrm{O}$ molecules and periodic 
boundary conditions were applied. The excess charge of the systems was compensated by a uniform background charge ${ }^{29}$ instead of adding counterions explicitly. After equilibrating the system for $\sim 2$ ps at $300 \mathrm{~K}$, the trajectories were collected for at least $40 \mathrm{ps}$. All the simulations were done in a $(N V T)$ canonical ensemble using a Nosé-Hoover thermostat ${ }^{30,31}$ to control the temperature. Additionally, the trajectories for solvated $\mathrm{Li}^{+}, \mathrm{Na}^{+}$, and $\mathrm{K}^{+}$, reported in Ref. 10 were re-analyzed whenever needed to support the discussion.

Figure 1 shows our computed radial distribution functions (RDFs) $g_{\mathrm{MO}}(r)$ and $g_{\mathrm{MH}}(r)$ for $\mathrm{M}=\mathrm{Rb}$ and Cs in ambient liquid water along with those for lighter alkali ions ${ }^{10}$ for comparison. The position of the first peak of $g_{\mathrm{MO}}(r)$ and $g_{\mathrm{MH}}(r)$ shifts steadily to the right and its height decreases with increasing alkali-ions atomic number, in remarkable agreement with reported experiments. ${ }^{33,34}$ Simultaneously, the second peak of $g_{\mathrm{CsO}}(r)$ almost disappears, in agreement with known experimental results, ${ }^{32}$ whereas the corresponding peak for $\mathrm{Rb}^{+}$remains visible, indicating that the interaction between $\mathrm{Cs}^{+}$and $\mathrm{H}_{2} \mathrm{O}$ molecules is likely to be exceptionally short-ranged.

The location of the first minimum of $g_{\mathrm{MO}}(r)$, which is normally used to evaluate the coordination number $(\mathrm{CN})$, cannot be unambiguously determined for both ions, as seen in Fig. 1. Therefore, hydration water molecules are identified in this work by adopting three different geometrical cutoff distances between each cation and the $\mathrm{O}$ atoms of solvating water molecules around in the range after the first peak where the RDF decreases and flattens. These distances were 3.7, 3.8, and 3.9 $\AA$ for $\mathrm{Rb}^{+}$and 3.8, 3.9, and $4.0 \AA$ for $\mathrm{Cs}^{+}$ and the corresponding distributions of the instantaneous $\mathrm{CNs}$ for $\mathrm{Rb}^{+}$and $\mathrm{Cs}^{+}$are shown in Fig. 2. We found that the in-

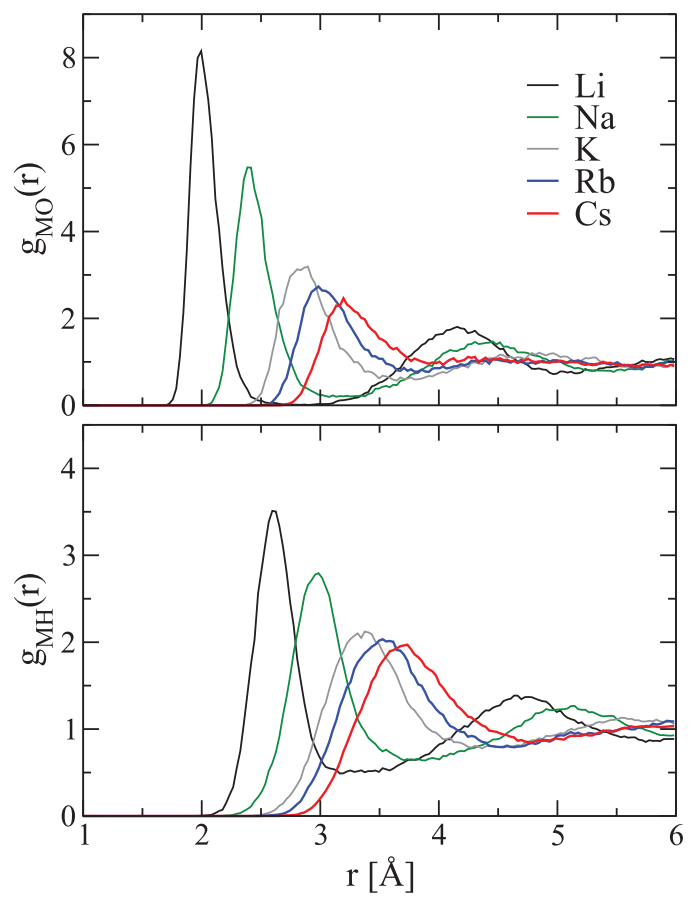

FIG. 1. Radial distribution functions $g_{\mathrm{MO}}(r)$ (upper) and $g_{\mathrm{MH}}(r)$ (lower) for $\mathrm{M}=\mathrm{Rb}$ and $\mathrm{Cs}$ in liquid water. For comparison, $g_{\mathrm{MO}}(r)$ and $g_{\mathrm{MH}}(r)$ of lighter alkali ions from Ref. 10 are also included in each panel.

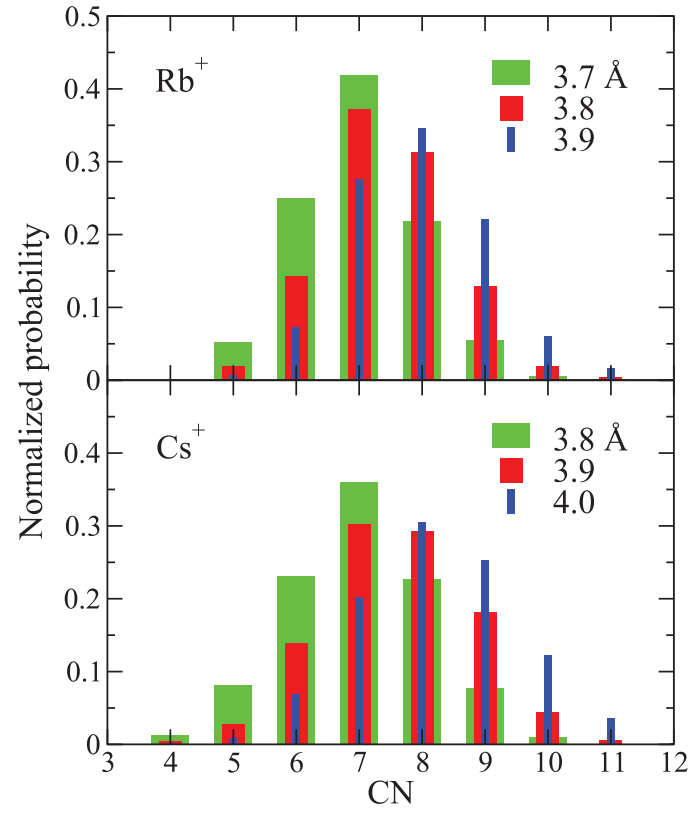

FIG. 2. Dependence of the instantaneous coordination numbers (CNs) of $\mathrm{Rb}^{+}$(upper) and $\mathrm{Cs}^{+}$(lower) on the choice of the cut-off distance (see text for details).

stantaneous $\mathrm{CNs}$ of $\mathrm{Cs}^{+}$show a much broader distribution in comparison with other alkali ions. Interestingly, even the tetra-coordination, which is missing for $\mathrm{K}^{+}$and $\mathrm{Rb}^{+}$, is reobserved for $\mathrm{Cs}^{+}$, suggesting that the hydration ability of $\mathrm{Cs}^{+}$ is significantly weaker than that of the other alkali ions. The averaged $\mathrm{CN}$ estimated as $7.0 \sim 8.0(7.0 \sim 8.2)$ for $\mathrm{Rb}^{+}$ $\left(\mathrm{Cs}^{+}\right)$falls into the available experimental data ranging from 6 to $8.4,6,27$

The distribution of the tilt angle $\psi$ is shown in Fig. $3 ;^{35}$ here $\psi$ is defined as sketched in the inset, namely the angle between the dipole vector of a hydrating $\mathrm{H}_{2} \mathrm{O}$ molecule and the vector pointing from the $\mathrm{O}$ atom of $\mathrm{H}_{2} \mathrm{O}$ to the cation. This angle is determined by a subtle balance between the ionwater interactions and the $\mathrm{H}$-bonds formed by $\mathrm{H}_{2} \mathrm{O}$ molecules belonging to the first and to the second solvation shells. The tendency of $\psi$ to decrease from $\mathrm{Li}^{+}$to $\mathrm{K}^{+}$, noticed in previous works, still holds for $\mathrm{Rb}^{+}$and $\mathrm{Cs}^{+}$, and its average value is $121^{\circ}$ and $119^{\circ}$ for $\mathrm{Rb}^{+}$and $\mathrm{Cs}^{+}$, respectively.

The average molecular dipole moment $\mu$ of $\mathrm{H}_{2} \mathrm{O}$ molecules in the first hydration shell of the alkali ions was computed via maximally localized Wannier function centers (WFCs) ${ }^{36,37}$ (Fig. 4). We found that $\mu$ of hydration waters decreases going from $\mathrm{Li}^{+}$to $\mathrm{K}^{+}$, but increases slightly going from $\mathrm{K}^{+}$to $\mathrm{Cs}^{+}$. This increase is accompanied by the increase of acceptor $\mathrm{H}$ bonds of hydration water molecules at the border of the first hydration shell of heavy alkali ions. In fact, the average number of acceptor $\mathrm{H}$ bonds, counted based on the local geometry, ${ }^{11}$ turns out to be $1.06,1.14$, and 1.18 when a cutoff distance of 3.7, 3.8, and $3.9 \AA$ is adopted for $\mathrm{K}^{+}, \mathrm{Rb}^{+}$, and $\mathrm{Cs}^{+}$, respectively. The further increase of cutoff distance by $0.1 \AA$ leads to the increase of the number of acceptor $\mathrm{H}$ bonds per hydration $\mathrm{H}_{2} \mathrm{O}$ molecule by 0.03 on average while keeping the number of donor $\mathrm{H}$ bonds constant. Considering that a large amount of hydration waters having $\psi<90^{\circ}$ are 


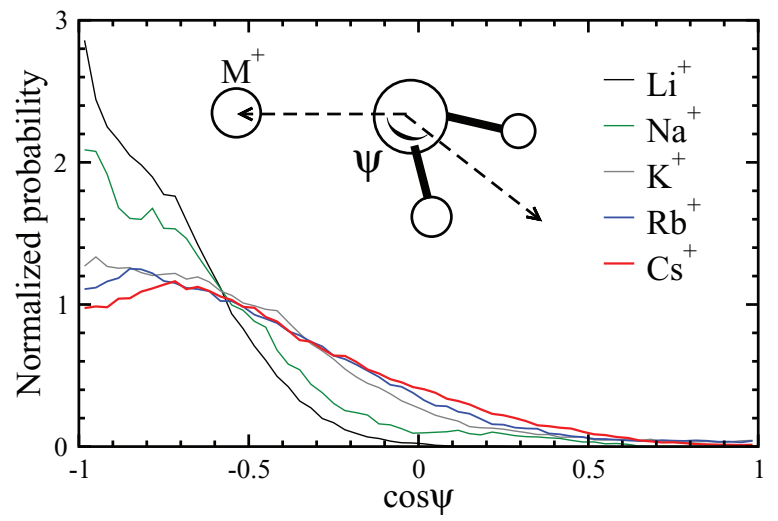

FIG. 3. Distribution of the cosine of the tilt angle $\psi$ between the dipole vector of hydration water molecules and the vector pointing from the $\mathrm{O}$ atom of water to the alkali ion. For comparison, distributions for the light alkali ions of Ref. 10 are also shown.

also observed for $\mathrm{K}^{+}, \mathrm{Rb}^{+}$, and $\mathrm{Cs}^{+}$and that their population increases in this order, the formation of the acceptor $\mathrm{H}$ bonds at the border of the solvation shell is expected to result in an enhancement of water exchange reactions. Indeed, our estimated residence time of hydration $\mathrm{H}_{2} \mathrm{O}$ molecules for $\mathrm{Rb}^{+}$ and $\mathrm{Cs}^{+}$is about half of the corresponding estimate for $\mathrm{Na}^{+}$ and $\mathrm{K}^{+} .{ }^{38}$ This indicates that water exchange occurs more frequently for these heavier ions than for the lighter ones.

According to the Pearson's hard and soft acids and bases (HSAB) concept, ${ }^{39}$ alkali ions are considered typical hard acids characterized by low polarizability. This is true for $\mathrm{Li}^{+}$ and $\mathrm{Na}^{+}$, for which we estimated a net dipole moment negligibly small. ${ }^{40}$ However, as shown in the inset of Fig. 5, heavy alkali ions, in water, have a net dipole moment reaching 0.4 , 0.6, and 0.8 Debye for $\mathrm{K}^{+}, \mathrm{Rb}^{+}$, and $\mathrm{Cs}^{+}$, respectively. This is mainly due to polarizable shallow core orbitals, which are included explicitly in our plane-wave pseudopotential approach. If the net dipole moment is not vanishing, as in the cases indicated above, the hydration shell is prone to variations and, as a consequence, the ion-water complex can become more stable. A special (and extreme) case is represented by surface solvation which can be realized depending on a delicate bal-

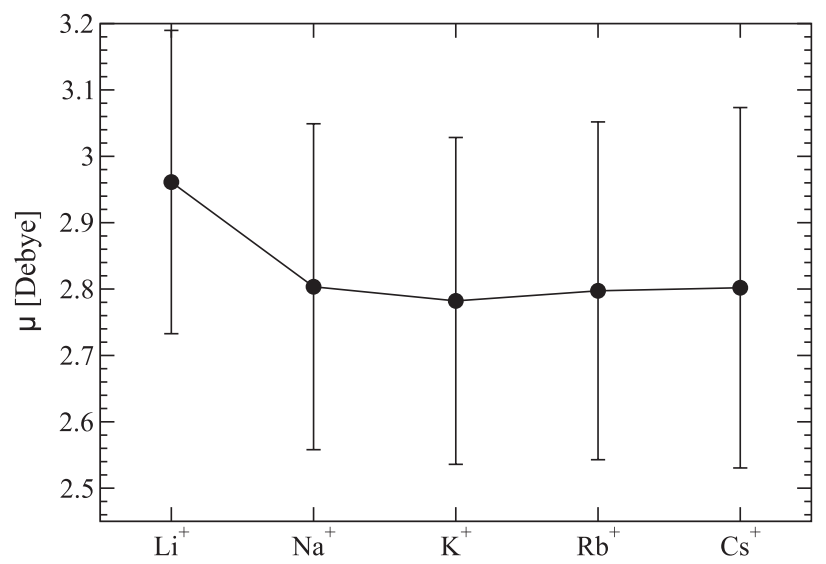

FIG. 4. Average molecular dipole moment $\mu$ of water molecules belonging to the first hydration shell computed from Wannier function centers (WFCs).

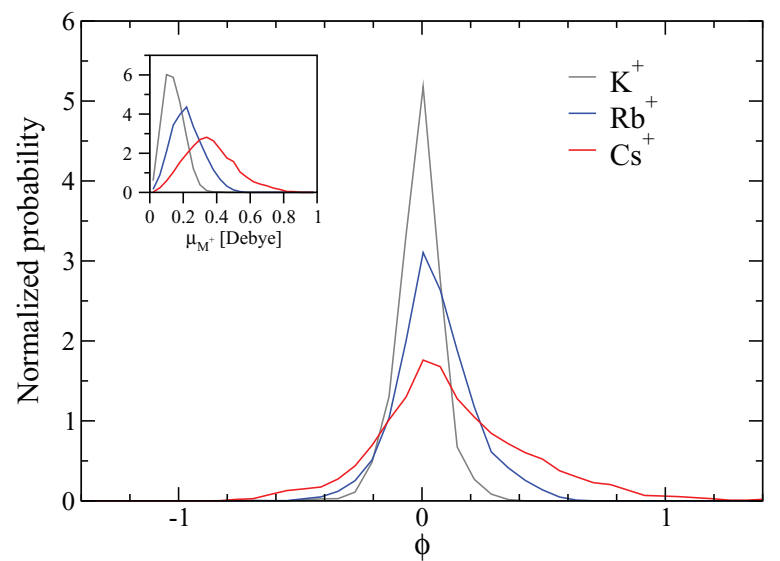

FIG. 5. Distribution of the correlation function $\phi$ between dipole vectors of ions $\boldsymbol{\mu}_{\mathrm{M}^{+}}$and the vector $\boldsymbol{R}_{\text {cage }}$ defined as in the text. The inset shows the distribution of $\mu_{\mathrm{M}^{+}}$for $\mathrm{K}^{+}, \mathrm{Rb}^{+}$, and $\mathrm{Cs}^{+}$in solution calculated from WFCs.

ance among the stabilization of the first solvation shell, the destabilization of the second shell, and longer distance interactions. This subtle interplay among different factors can determine whether or not an asymmetric hydration shell, such as the one realized at surfaces, can appear.

To examine the possibilities of forming such an asymmetric shell for alkali ions, we introduce a vector given by $\boldsymbol{R}_{\text {cage }}=\sum_{i=1}^{\mathrm{CN}}\left(\boldsymbol{R}_{\mathrm{O}_{i}}-\boldsymbol{R}_{\mathrm{M}^{+}}\right) /\left|\boldsymbol{R}_{\mathrm{O}_{i}}-\boldsymbol{R}_{\mathrm{M}^{+}}\right|$. Here the sum for the oxygen positions $\boldsymbol{R}_{\mathrm{O}_{i}}$ runs over the $\mathrm{O}$ atoms in the solvation shell at a given moment for a cation located at $\boldsymbol{R}_{\mathrm{M}^{+}}$. The distribution of the modulus $\left|\boldsymbol{R}_{\text {cage }}\right|$ (not shown) indicates that for $\mathrm{Li}^{+}$and $\mathrm{Na}^{+}$the hydration shell is highly symmetric $\left(\left\langle\left|\boldsymbol{R}_{\text {cage }}\right|\right\rangle \leq 0.5\right)$ while for heavy alkali ions it tends to be asymmetric. In particular for $\mathrm{Cs}^{+}$the predominant structure is indeed asymmetric $\left(\left\langle\left|\boldsymbol{R}_{\text {cage }}\right|\right\rangle \simeq 1.2\right)$.

An order parameter $\phi$ can be defined as $\phi=\boldsymbol{\mu}_{\mathrm{M}^{+}} \cdot \boldsymbol{R}_{\text {cage }}$ which rationalizes the possible correlation between a net cation dipole $\boldsymbol{\mu}_{\mathrm{M}^{+}}$and the asymmetry of the solvation shell measured with respect to the reference $\boldsymbol{R}_{\text {cage. }}$. The strong asymmetric distribution of $\phi$ observed for $\mathrm{Cs}^{+}$(Fig. 5) evidences that the polarization of $\mathrm{Cs}^{+}$is strongly correlated with its asymmetric first hydration shell, thus making $\mathrm{Cs}^{+}$a peculiar case among the other alkali ions.

Summarizing, we studied hydration structure and polarization of $\mathrm{Rb}^{+}$and $\mathrm{Cs}^{+}$in liquid water at ambient conditions by first principles molecular dynamics. Our systematic analysis of the hydration structure and the related electronic structure peculiarities, based on maximally localized Wannier functions and their centers, revealed close relationships among molecular orientation, H-bond formation, and polarization of water molecules around the hydrated cations. Furthermore, we found that the polarization of heavy alkali ions, especially $\mathrm{Cs}^{+}$, tends to stabilize an asymmetric hydration structure. All these findings indicate rather clearly that $\mathrm{Cs}^{+}$possesses an intrinsic instability toward forming surface solvation states which would be a key step to adsorption of hydrated $\mathrm{Cs}^{+}$and in this peculiarity, along with the specific $\mathrm{Cs}^{+}$-clay interactions, resides the difficulty in the extraction of ${ }^{137} \mathrm{Cs}$ from clay minerals. 
The calculations reported in this paper were performed at the JAEA supercomputer facility. We wish to dedicate this paper to the victims of Fukushima.

${ }^{1}$ B. E. Conway, Ionic Hydration in Chemistry and Biophysics, Studies in Physical and Theoretical Chemistry 12 (Elsevier, Amsterdam, 1981).

${ }^{2}$ Y. Marcus, Ion Solvation (Wiley, Chichester, 1986).

${ }^{3}$ J. Burgess, Metal Ions in Solution (Ellis Horwood, New York, 1978).

${ }^{4}$ D. T. Richens, The Chemistry of Aqua Ions (Wiley, Chichester, 1997).

${ }^{5}$ Y. Marcus, Chem. Rev. 88, 1475 (1988).

${ }^{6}$ H. Ohtaki and T. Radnai, Chem. Rev. 93, 1157 (1993).

${ }^{7}$ J. Smith, Nature (London) 472, 7 (2011).

${ }^{8}$ G. Brumfiel, Nature (London) 478, 435 (2011).

${ }^{9}$ W. Stumm and J. J. Morgan, Aquatic Chemistry, 3rd ed. (Wiley, New York, 1996).

${ }^{10}$ T. Ikeda, M. Boero, and K. Terakura, J. Chem. Phys. 126, 034501 (2007).

${ }^{11}$ R. Scipioni, D. A. Schmidt, and M. Boero, J. Chem. Phys. 130, 024502 (2009).

${ }^{12}$ D. A. Schmidt, R. Scipioni, and M. Boero, J. Phys. Chem. A 113, 7725 (2009).

${ }^{13}$ R. Car and M. Parrinello, Phys. Rev. Lett. 55, 2471 (1985).

${ }^{14}$ CPMD, Copyright IBM Corp 1990-2008, MPI für Festkörperforshung Stuttgart 1997-2001, see http://www.cpmd.org/.

${ }^{15}$ W. Kohn and L. J. Sham, Phys. Rev. 140, A1133 (1965).

${ }^{16}$ F. A. Hamprecht, A. J. Cohen, D. J. Tozer, and N. C. Handy, J. Chem. Phys. 109, 6264 (1998).

${ }^{17}$ T. Ikeda, M. Boero, and K. Terakura, J. Chem. Phys. 127, 074503 (2007).

${ }^{18}$ T. Ikeda, M. Hirata, and T. Kimura, J. Chem. Phys. 122, 244507 (2005).

${ }^{19}$ J. M. Park and M. Boero J. Phys. Chem. B 114, 11102 (2010).

${ }^{20}$ See M. Sprik, J. Hutter, and M. Parrinello, J. Chem. Phys. 105, 1142 (1996).
${ }^{21}$ N. Troullier and J. L. Martins, Phys. Rev. B 43, 1993 (1991).

${ }^{22}$ S. Goedecker, M. Teter, and J. Hutter, Phys. Rev. B 54, 1703 (1996).

${ }^{23}$ C. Hartwigsen, S. Goedecker, and J. Hutter, Phys. Rev. B 58, 3641 (1998).

${ }^{24}$ J. C. Grossman, E. Schwegler, E. W. Draeger, F. Gygi, and G. Galli, J. Chem. Phys. 120, 300 (2004).

${ }^{25}$ E. Schwegler, J. C. Grossman, F. Gygi, and G. Galli, J. Chem. Phys. 121, 5400 (2004).

${ }^{26}$ F. A. Bornemann and C. Schütte, Numer. Math. 78, 359 (1998).

${ }^{27}$ M. J. San-Román, J. Hernández-Cobos, H. Saint-Martin, and I. OrtegaBlake, Theor. Chem. Acc. 126, 197 (2010).

${ }^{28}$ M. Kołaski, H. M. Lee, Y. C. Choi, K. S. Kim, P. Tarakeshwar, D. J. Miller, and J. M. Lisy, J. Chem. Phys. 126, 074302 (2007).

${ }^{29}$ G. Makov and M. C. Payne, Phys. Rev. B 51, 4014 (1995).

${ }^{30}$ S. Nosé, Mol. Phys. 52, 255 (1984); S. Nosé, J. Chem. Phys. 81, 511 (1984).

${ }^{31}$ W. G. Hoover, Phys. Rev. A 31, 1695 (1985).

${ }^{32}$ S. Ramos, G. W. Neilson, A. C. Barnes, and P. Buchanan, J. Chem. Phys. 123, 214501 (2005).

${ }^{33}$ P. R. Smirnov and V. N. Trostin, Russ. J. Gen. Chem. 77, 2101 (2007).

${ }^{34}$ J. Mähler and I. Persson, Inorg. Chem. 51, 425 (2012).

${ }^{35}$ For subsequent analyses of hydration of $\mathrm{Rb}^{+}$and $\mathrm{Cs}^{+}$, we used the cutoff distance of 3.8 and $3.9 \AA$, respectively, unless otherwise mentioned.

${ }^{36}$ P. L. Silvestrelli and M. Parrinello, Phys. Rev. Lett. 82, 3308 (1999).

${ }^{37}$ N. Marzari and D. Vanderbilt, Phys. Rev. B 56, 12847 (1997).

${ }^{38}$ The residence time of water molecules in the first shell, estimated according to the prescrition of Impey et al. [J. Phys. Chem. 87, 5071 (1983)], are 7 and 9 ps for $\mathrm{Rb}^{+}$and $\mathrm{Cs}^{+}$, respectively.

${ }^{39}$ R. G. Pearson, J. Am. Chem. Soc. 85, 3533 (1963).

${ }^{40}$ J. J. Molina, S. Lectez, S. Tazi, M. Salanne, J.-F. Dufrêche, J. Roques, E. Simoni, P. A. Madden, and P. Turq, J. Chem. Phys. 134, 014511 (2011) 